\title{
EFFECT OF FOREIGN PROTEIN ON THE KIDNEY
}

\author{
E. T. BELL AND T. B. HARTZELL \\ From the Department of Pathology, University of Minnesota, and the National Dental \\ Research Association
}

It is known that some persons develop symptoms of anaphylactic character after eating certain foods, of which shellfish, egg-white, and certain fruits are familiar examples. It is believed by many that asthma, urticaria and hay-fever have their origin largely in a condition of hypersensitiveness to special foreign proteins. The symptoms produced when a foreign protein is introduced into a sensitized animal are usually referred to a hypothetical substance- "anaphylatoxin"-formed by the action of antibody on antigen. There are reports in the literature to the effect that anatomic changes in the tissues occur in acute anaphylaxis, and Longcope ${ }^{1}$. has advanced the theory that repeated anaphylactic shock may be responsible for such chronic diseases as nephritis. By a series of animal experiments Longcope believes that he has shown that repeated nonfatal poisoning with foreign protein is responsible for some cases of chronic nephritis, cirrhosis of the liver, and chronic myocarditis. Our interest in the etiology of chronic nephritis led us to repeat some of his experiments.

\section{ANATOMIC CHANGES IN ACUTE ANAPHYLAXIS}

Gay and Southard ${ }^{2}$ described hemorrhages and areas of fatty degeneration in guinea-pigs that died in acute anaphylactic shock and in those killed subsequent to severe shock. The hemorrhages were widely distributed, but were most commonly found in the mucosa of the stomach and in the lungs. Areas of fatty degeneration were found in capillary endothelium, in the mucosa of the stomach, and in the cardiac and voluntary muscle. The hemorrhages were interpreted as the result of the degeneration of the capillary endothelium. But Anderson and Rosenau ${ }^{3}$ in repeating this work could find no evidence

Received for publication Feb. 2, 1918.

1 Jour. Exper. Med., 1913, 18, p. 678; 1915, 22, p. 793; Trans. Assn. Am. Phys., 1914, 29 , p. 49.

2 Jour. Med. Research, 1907, 16, p. 143; 1908, 18, p. 17.

3 Jour. Med. Research, 1908, 19, p. 57. 
of fatty degeneration. They did not find the hemorrhages so constantly and seem inclined to interpret them, when present, as due to the violent struggling of the animal during shock rather than to the action of the protein.

It is known also that fatty degeneration does not occur suddenly. Usually several days are required for a cell to become definitely fatty, and very little fat accumulates earlier than 48 hours after the injury. It is inconceivable that this change could occur within a few minutes or even within a few hours. Again the normal variations in the fat content of voluntary muscle fibers are so great that one can seldom speak with assurance of a fatty degeneration in this tissue. These considerations lend strong support to the negative findings of Anderson and Rosenau.

The Arthus ${ }^{4}$ phenomenon seems to lend experimental support to the view that foreign protein may produce local anatomic changes when introduced into a sensitive animal.

Longcope ${ }^{5}$ studied the effects of repeated injections of foreign protein in guinea-pigs, rabbits, dogs and cats. Small sensitizing doses of horse serum or egg-white were given 20-25 days before the secondary injections were begun. The secondary injections were spaced and regulated with the object of producing shock a number of times. Some of the experiments lasted as long as 3 months, during which time as many as 15 injections were given. Not all injections resulted in anaphylactic symptoms and many of the reactions were recorded as slight.

Changes were found in the kidneys in 23 of 29 rabbits, and in a large proportion of the other animals experimented on. In the animals that died acutely diffuse degenerative changes were found. In those that lived longer, extensive round cell infiltration was the most striking change. Dense fibrous rays with atrophic tubules were found in 6 rabbits. Six rabbits showed normal kidneys at necropsy. Portal cirrhosis of the liver and areas of chronic myocarditis were also found frequently.

In a more recent paper Longcope ${ }^{6}$ found that similar though less marked renal lesions may be produced by a single large injection of foreign protein, or by several injections in which a different protein was used each time. All the sensitized animals developed nephritis,

4 Arch. internat. de Physiol., 1908-9, 7, p. 471.

J Jour. Exper. Med., 1913, 18, p. 678.

a Ibid., 1915, 22, p. 793. 
but of those that received a single large injection, or several different proteins, only one-third developed nephritis. He concluded that foreign protein is itself toxic and may produce nephritis, but that previous sensitization increases the extent, severity and constancy of the lesions.

The lesions described by Longcope correspond in all respects with those of ordinary spontaneous nephritis. As controls, 24 supposedly normal rabbits were studied, and in 4 of these a few small areas of round cell infiltration were found similar to those occurring in the experimental animals. The argument that the lesions were produced by the foreign protein because they were more extensive and severe than in the controls has no value, since advanced stages of spontaneous nephritis are of common occurrence. The controls apparently did not have the same opportunity to contract an infection, since there is no mention of their having been kept under the same conditions and for the same length of time as the experimental animals. It is also to be noted that some of the animals may have had nephritis at the outset of the experiment, since it is admitted that animals with a faint trace of albumin were used. Again, there was no control at all for the healed stages of nephritis, of which 6 cases are mentioned and attributed to the action of the protein, since these do not cause albuminuria. No cultures were taken from the kidneys to determine whether the nephritis was due to infective or to chemical irritants.

Three of Longcope's experiments were controlled somewhat better. Three dogs were operated on, and a portion of one kidney from each was removed for examination before the secondary inoculations were begun. The excised portions of the kidneys were found normal, but each animal developed nephritis after a number of injections of protein subsequent to the operation. But no cultures were made from these kidneys, and a renal infection was therefore not excluded.

The uncertainty of the results of Longcope's work is indicated further by his attributing the ordinary cirrhosis of the liver of rabbits to the action of the protein. This is an unusually common lesion. It occurs in fully three-fourths of adult rabbits and is probably due to bacterial infection. All who have studied rabbit livers extensively recognize this as a spontaneous lesion.

Longcope's conclusions were confirmed by Boughton ${ }^{7}$ to the extent that repeated injections of foreign protein may produce renal lesions.

' Jour. Immunology, 1916, 1, p. 105. 
Boughton produced repeated anaphylactic shock in guinea-pigs, using beef serum and egg-white as antigens. Some of the animals received as many as 19 secondary injections, and the average number of injections was 9. The average duration of the experiments was 4 months, but some animals were under experimentation as long as 8 months. The urine was not examined systematically. Twenty-three guineapigs were used. Degeneration, desquamation, and necrosis of the tubular epithelium was the most striking change noted and was seen in all cases. Round cell infiltration was found in nearly all cases, though it was never vary marked. There was no distinct scar formation.

Six controls were given injections of protein so spaced as to avoid the production of anaphylaxis. Tubular injury was much less marked than in the sensitized animals. Areas of round cell infiltration were also seen, but these were only one-tenth to one-fifteenth as frequent as in the sensitized animals.

All injections were given intraperitoneally and ranged as high as $10 \mathrm{cc}$ (undiluted serum, or $50 \%$ solution of egg-white). The kidney lesions were regarded as subacute rather than chronic.

Boughton seems to conclude that foreign protein produces some renal injury when injected without the production of anaphylactic shock, and rather severe injury when this phenomenon is repeatedly produced. The tubular injury was the most prominent lesion, and the production of a typical chronic nephritis is apparently not claimed. As regards the interpretation of the round cell infiltration, the sources of error are the same as in Longcope's experiments.

\section{EXPERIMENTS}

The purpose of our investigation was to determine whether chronic nephritis can be experimentally produced in rabbits by a foreign protein. Repeated shock was produced in a few animals, but in most of the experiments no attempt was made to produce anaphylaxis.

Special effort has been made to eliminate various sources of error. All rabbits that had albuminuria were discarded. In order to eliminate the occasional inactive cases of spontaneous nephritis, rabbits with normal urine were operated on with aseptic precautions under ether anesthesia in order to expose and examine the surface of the left kidney. All except early cases of nephritis may be recognized by the 
presence of spots and pits on the surface of the kidney. Both kidneys are nearly always involved to about the same extent, so that it is not necessary to examine the right kidney. About one week after the operation, the experiment was begun in case the wound was completely healed and the urine free from albumin. Ascitic fluid, egg-albumin, and sheep serum were used. Injections were made intravenously except as otherwise indicated.

\section{Ascitic Fluid with Anaphylaxis}

Rabbit 25.--Weight $2,000 \mathrm{gm}$. July 3: Test for albumin negative. Left kidney exposed and found normal.

July 12,17, 24:1 c c sensitizing injections of ascitic fluid.

Sept. 10: 1 c c, moderate anaphylactic symptoms.

Sept. $21: 0.5$ c c, slight symptoms.

Oct. 5: $0.25 \mathrm{cc}$, slight symptoms.

Oct. $15: 0.5 \mathrm{cc}$, slight symptoms.

Oct. $25: 0.5 \mathrm{c} \mathrm{c}$, moderate symptoms.

Nov. 5: $0.5 \mathrm{cc}$, moderate symptoms.

Nov. $15: 1 \mathrm{cc}$, severe symptoms.

Nov. 21: Found dead.

Tests for albumin negative, Sept. 10, 25, Oct. 11. Trace of albumin, Nov. 7. Seven secondary injections were given over a period of 71 days. At necropsy the kidneys showed no gross changes. Microscopically an occasional small focus of lymphocytes was found deep in the cortex.

Rabbit 35.-Weight 1,885 gm. Sept. 11: Test for albumin negative. Left kidney exposed and found normal.

Oct. 5: $0.25 \mathrm{cc}$, ascitic fluid.

Oct. $15: 0.5 \mathrm{c} \mathrm{c}$, slight symptoms.

Oct. $25: 0.5$ c c, moderate symptoms.

Nov. 5: $1 \mathrm{c} \mathrm{c}$, moderate symptoms.

Nov. 15: $1 \mathrm{cc}$, slight symptoms.

Nov. 26: 2 c c, slight symptoms.

Dec. 6: $2.5 \mathrm{c} \mathrm{c}$, moderate symptoms.

Dec. $17: 2.5 \mathrm{cc}$, slight symptoms.

Dec. $27: 2.5 \mathrm{cc}$, slight symptoms.

Jan. 10: Killed.

Tests for albumin negative, Nov. 8; positive, Jan. 4 and 10. On Dec. 5, the serum from this rabbit gave a strong precipitin test with human blood serum. Eight secondary injections were given over a period of 87 days. At necropsy the kidneys showed no gross changes. Microscopically an occasional small lymphocytic focus was found in the cortex.

Rabbit 33.-Weight $2,320 \mathrm{gm}$. July 18: Test for albumin negative. Left kidney exposed and found normal.

Sept. 10: $0.5 \mathrm{cc}$, ascitic fluid.

Sept. $21: 0.5 \mathrm{cc}$, severe symptoms.

Oct. 5: $0.25 \mathrm{cc}$, slight symptoms.

Oct.. 15: $0.25 \mathrm{cc}$, slight symptoms.

Oct. $25: 0.5 \mathrm{cc}$, moderate symptoms.

Nov. $5: 0.5 \mathrm{c} \mathrm{c}$, moderate symptoms. 
Nov. 15: $1 \mathrm{cc}$, slight symptoms.

Nov. $26: 2 \mathrm{cc}$, slight symptoms.

Dec. $6: 2.5 \mathrm{cc}$, slight symptoms.

Dec. 17: Found dead.

Tests for albumin negative, Sept. 10, 25, Oct. 11, Nov. 7. On Dec. 4 the serum from this rabbit gave no precipitate with human blood serum. Eight secondary injections were given over a period of 87 days. The kidneys showed no lesions grossly or microscopically.

\section{Ascitic Fluid Without Anaphylaxis}

Rabbit 32.-Weight $2,320 \mathrm{gm}$. Oct. 2: Test for albumin negative.

Oct. 6: Left kidney exposed and found normal.

One c c ascitic fluid, Oct. 16, 23 .

Two c c, Oct. 27, Nov. 1, 6, 12, 17.

Three c c, Nov. 22.

3.5 c c, Nov. 27 , Dec. $3,8,13,18,22,26,31$.

Tests for albumin negative, Oct. 13, Nov. 9, 22, Dec. 13; positive, Jan. 3.

Killed, Jan. 3. 16 injections were given over a period of 79 days.

No gross changes found in the kidneys. Microscopically a few small lymphocytic foci found in the cortex.

Rabbit 54.-Weight 2,105 gm. Jan. 28: Test for albumin negative.

Jan. 31 : Left kidney exposed and found normal. Injections of ascitic fluid as follows: Feb. 11, 0.5 c c; Feb. 19, 0.75 c c; Feb. 23, 1 c c; Feb. 28, 0.75 c c; Mar. 5, $0.75 \mathrm{cc}$; Mar. 12, 1 c c; Mar. 19, 1 c c; Mar. 26, 1 c c; Apr. 3, 1 c c. Killed, Apr. 8.

Albumin tests negative Feb. 14, 21, Mar. 2, 26, Apr. 5. Nine injections were given over a period of 56 days. No gross or microscopic lesions found in the kidneys.

Rabbit 65.-Weight 2,170 gm. Dec. 6: Albumin test negative. Left kidney exposed and found normal. Injections of ascitic fluid as follows: Dec. 22, 0.25 c c; Dec. 26, 0.5 c c; Dec. 31, 0.5 c c; Jan. 11, 0.75 c c; Jan. 22, 1 c c; Feb. 5, 1.5 c c (slight shock); Feb. 11, 1.5 c c; Feb. 19, 1.5 c c; Feb. 23, 2 c c; Feb. 28, $1 \mathrm{cc}$; Mar. 5, $1 \mathrm{cc}$; Mar. 12, $1 \mathrm{cc}$.

Killed Mar. 18. Twelve injections were given over a period of 86 days. Albumin tests negative, Dec. 14, Jan. 3, 30, Mar. 2. No gross or microscopic lesions found in the kidneys.

\section{Experiments with EgG-Albumin}

Rabbit 44.-Weight 2,250 gm. Jan. 3: Test for albumin negative.

Jan. 5: Left kidney exposed and found normal.

Injections of a $1 \%$ solution of egg-albumin as follows: Jan. 15, $0.25 \mathrm{cc}$; Jan. 22, 0.5 c c; Jan. 26, $0.75 \mathrm{c} \mathrm{c}$; Jan. 30, $1 \mathrm{c} \mathrm{c}$; Feb. 5, $1.5 \mathrm{c} \mathrm{c}$; Feb. 11, $2 \mathrm{cc}$ of a $50 \%$ solution of egg-white intraperitoneally. Death, Feb. 11.

Albumin tests negative, Jan. 15, 25, 30. At necropsy the kidneys were found slightly swollen and moist. There was precipitated albumin in the capsular spaces and in the lumina of the convoluted tubules. Whether this albumin was excreted egg-albumin or serum-albumin was not determined. There were no areas of lymphocytic infiltration.

Rabbit 50.-Weight 1,850 gm. Feb. 28: Albumin test negative. Left kidney exposed and found normal. Intraperitoneal injections of a $50 \%$ solution of 
egg-albumin as follows: Mar. 14, $0.5 \mathrm{c} \mathrm{c}$; Mar. 19, 1 c c; Mar. 26, 1 c c; Apr. 3, 1 c c; Apr. 8, 1 c c; Apr. 11, 1 c c; Apr. 16, 1 c c. Death, Apr. 19.

Albumin tests negative, Mar. 19, Apr. 5, 19. Seven injections were given during a period of 36 days. No gross or microscopic lesions found in the kidneys.

Rabbit 60 .-Weight $1,560 \mathrm{gm}$. Dec. 5: Test for albumin negative.

Dec. 6: Left kidney exposed and found normal.

One c c of a $1 \%$ solution of egg-albumin was injected intravenously on the following dates: Dec. 20, 26, 31, Jan. 11, 22, and Feb. 5. One c c of a $50 \%$ solution of egg-white was injected intraperitoneally on Feb. 11, 19, 23, 25, and Mar. 5. Death, Mar. 9.

Albumin tests negative Dec. 14, Jan. 5, 15, 30, and Feb. 25. Trace of albumin, Jan. 25.

No gross changes found in the kidneys. Microscopically an occasional cast was observed, but there were no areas of lymphocytic infiltration.

\section{Experiments with Sheep Serum}

Rabbit 38.-Weight 1,950 gm. Dec. 3: Albumin test negative. Dec. 6: Left kidney exposed and found normal. Injections of sheep serum, diluted with equal volume of salt solution, as follows : Jan. 19, $0.5 \mathrm{c} \mathrm{c}$; Jan. 24, $0.75 \mathrm{c} \mathrm{c}$; Jan. 29, 1 c c; Feb. 5, 1.5 c c. Found dead, Feb. 6.

Albumin tests negative, Jan. 28, Feb. 1. The kidneys were swollen and cloudy. Precipitated albumin was found in the lumens of some of the convoluted tubules. There were no areas of lymphocytic infiltration.

Rabbit 42.-Weight 1,750 gm. Jan. 4: Test for albumin negative. Jan. 15 : Left kidney exposed and found normal. Jan. 19, $0.5 \mathrm{cc}$ of sheep serum. Jan. 22, found dead. Kidneys were cloudy. No microscopic examination.

Rabbit 53.-Weight 2,970 gm. Feb. 7: Left kichey exposed and found normal. Feb. 14: Test for albumin negative. Injections of sheep serum as follows: Feb. 19, 0.5 c c; Feb. 23, 0.75 c c; Feb. 28, 0.5 c c; Mar. 5, 0.5 c c; Mar. 12, 0.5 с с; Mar. 21, 1 с c; Mar. 26, 1 с с; Apr. 3, 1 с c. Apr. 4, found dead.

Albumin tests negative, Mar. 12, 21. Kidneys showed no gross changes. Microscopically precipitated albumin was found in the lumina of many of the tubules. There were. a few.casts. No areas of lymphocytic infiltration seen.

Rabbit 63.-Weight 1,860 gm. Feb. 7: Test for albumin negative. Left kidney exposed and found normal. Injections of sheep serum as follows: Feb. 19, 0.5 c c; Feb. 23, 0.75 c c; Feb. 28, 0.5 c c; Mar. 5, 0.5 c c; Mar. 12, 0.5 c c; Mar. 21, 1 c c; Mar. 26, 1 c c; Apr. 3, 1 c c; Apr. 4, found dead.

Albumin tests negative, Feb. 14, Mar. 21. Trace of albumin, Mar. 12. Several small pits were found on the surfaces of each kidney. Microscopically there were small areas of lymphocytic infiltration corresponding to the surface pits.

Rabbit 67 .-Weight $1,960 \mathrm{gm}$. Jan. 4: Test for albumin negative. Jan. 5: Left kidney exposed and found normal. Injections of sheep serum as follows: Jan. 19, 0.5 c c; Jan. 24, 0.75 c c; Feb. 5,1 c c; Feb. 19, 1 c c; Feb. 23, 1.5 c c; Feb. 28, 1 c c; Mar. 5, 1 c c; Mar. 12, 1 c c; Mar. 21, 1 c c; Mar. 26, 1 c c; Apr. 3, 1 c c; Apr. 11, 1 c c; Apr. 16, 1 c c; Apr. 18, 0.5 c c. Killed, Apr. 30.

Albumin tests negative, Jan. 17, 28, Feb. 25, Mar. 14; positive, Apr. 4, 18. A number of fairly deep pits were seen on the surfaces of the kidneys. Microscopically rather extensive lymphocytic infiltrations were found underlying the 
pits. The lesions correspond with those of a well advanced spontaneous nephritis.

Rabbit 113.-Weight 1,824 gm. May 14: Albumin test negative. Left kidney removed and found normal grossly and microscopically. Injections of sheep serum as follows: May 28, 0.5 c c; June 4, $0.75 \mathrm{cc}$; June 13, 2 c c; June 27, 2 c c; June 29, 1 c c; July 2, 2 c c. Killed, July 11. Loss of weight during experiment, $474 \mathrm{gm}$. Albumin tests positive, June 27 , July 2, 10. The right kidney was covered with pits. Rather large areas of lymphocytic infiltration were found deep in the cortex underlying the pits, the appearances being those of a spontaneous nephritis. Cultures from the kdney in dextrose broth gave a pure growth of a streptococcus.

Rabbit 103.-Weight 1,250 gm. May 2: No albumin. Left kidney exposed and found normal. Injections of sheep serum as follows: May 28, $0.5 \mathrm{cc}$; June 4, 0.75 c c; June $6,0.75$ c c; June 13, 2 c c; June 27,2 c c; June 29,1 c c; July $2,2 \mathrm{cc}$. Killed, July 6. No loss of weight. Albumin tests positive, June 27, July 3. The kidneys showed many fairly deep pits. Microscopically there were rather large areas of lymphocytic infiltration. The appearances were those of a spontaneous nephritis. Cultures from the kidneys taken immediately after death gave a pure growth of a streptococcus.

\section{DISCUSSION}

These experiments indicate that ascitic fluid does not injure the kidney of a rabbit when injected intravenously in moderate amounts. The occasional small foci of lymphocytes found at necropsy in the injected animals can hardly be attributed to the protein, since such lesions are commonly found in nonexperimental animals and were not excluded by the preliminary examination. Lesions of this size produce no changes on the kidney surface and seldom cause albuminuria.

The production of anaphylactic symptoms repeatedly did not cause nephritis. The symptoms were usually rather mild, but it is not possible to produce severe shock repeatedly. The animals either die or become refractory. The symptoms were apparently nearly as pronounced as in the experiments of Longcope and Boughton.

Egg-albumin in large doses seemed to cause a mild parenchymal injury of the kidney occasionally, but no chronic changes of any kind were produced. This protein seems to be only mildly toxic for rabbits.

Sheep serum, however, is evidently toxic for rabbits, especially in relatively large doses. Several animals died shortly after a single injection. It produces a tubular injury to the kidney, but this is not severe enough to cause the death of the animal. There is no evidence of special glomerular injury. Degenerative changes only were observed in the first three experiments; but during the summer inflammatory renal lesions were found in 4 rabbits. This is evidently the same 
lesion. which Longcope obtained. It undoubtedly developed during the course of the experiments, since it was not present at the beginning. It is similar in all respects to ordinary spontaneous nephritis. Like the spontaneous disease it would seem to be due to a bacterial infection, since pure cultures of streptococci were obtained from the kidneys of rabbits 113 and 103.

Our interpretation of these apparently positive results with sheep serum is, therefore, that the animals acquired an infection during the course of the experiments. This infection may have been contracted from other rabbits in the animal house, or it may have been introduced by the intravenous injections, as these were not made with complete aseptic precautions. In either case it is probable that the injury of the kidney by the sheep serum favored the development of the infection. The injured kidney may have been a locus minoris resistentiae.

If this interpretation be correct it may be said that injections of sheep serum cause nephritis in rabbits by injuring the kidney and giving opportunity for the lodgment and growth of bacteria which may gain access to the circulation; but this is not a nephritis due directly to the action of anaphylatoxin or foreign protein. It is conceivable that toxic foreign protein might have a similar effect in man, but it is unlikely that such proteins ever gain access to the circulation in such relatively large amounts as are used experimentally in animals. And finally, if we grant the possibility that toxic foreign protein may enter the circulation and injure the kidney, we would expect the development of lesions similar to those found in the rabbit. But the only human renal lesions comparable to spontaneous nephritis of the rabbit are the hematogenous infections, namely, abscess of the kidney, descending pyelonephritis, and acute interstitial nephritis. Aside from the arteriosclerotic kidney, nearly all cases of chronic nephritis in man are examples of chronic glomerulo nephritis which has no anatomic resemblance whatever to spontaneous nephritis in the rabbit. It is therefore clear that the experimental production of such a lesion as spontaneous nephritis does not throw any light on the etiology of chronic nephritis in man.

It is not improbable that the various experimenters, who believe they have produced a chronic nephritis experimentally with uranium, potassium chromate, foreign protein, streptococci, etc., have been dealing with a spontaneous nephritis which was contracted during the experiment and hastened in its development by renal irritants. But 
even if all these claims were true we still have made little progress in determining the etiology of chronic nephritis in man.

As regards the etiology of human chronic nephritis we share the view, now widely held, that streptococci are the main causative agents, and that they first produce acute glomerular lesions which in many instances heal, but in others pass into a chronic stage. The continuous escape of streptococci from a focal infection or through a diseased mucous membrane would theoretically favor the development of chronic glomerular lesions. But thus far no one has been able to furnish experimental proof to support this theory.

\section{SUMMARY}

Nontoxic foreign proteins, such as ascitic fluid, do not produce renal injury of any kind in the rabbit when injected intravenously in moderate amounts. The repeated production of anaphylactic symptoms with this protein likewise does not injure the kidney.

Egg-albumin may produce slight parenchymal injury when injected in large amounts, but usually it causes no damage.

Toxic foreign proteins such as sheep serum produce considerable parenchymal injury to the kidney which in some instances seems to lead to the rapid development of a renal infection comparable in all anatomic respects to the ordinary type of spontaneous nephritis. The fact that streptococci were isolated in pure culture from the kidneys of such animals shows that the lesion is essentially infective, and not the direct result of foreign protein or anaphylatoxin.

No lesion comparable to human chronic glomerulo-nephritis has been produced experimentally.

There is no experimental evidence that foreign protein is in any way responsible for chronic nephritis in man. 